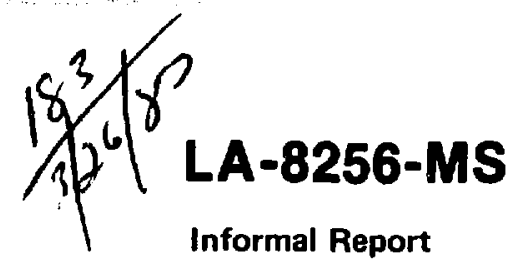

Informal Report

LA-8256-MS
DR.957

Informal Report

$\mid$

Light-Water Reactor Accident Classification

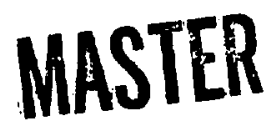




\title{
LA-8256-MS
}

informal Report

UC-78

Issued: February 1980

\section{Light-Water Reactor Accident Classification}

\author{
Beverly W. Washburn
}
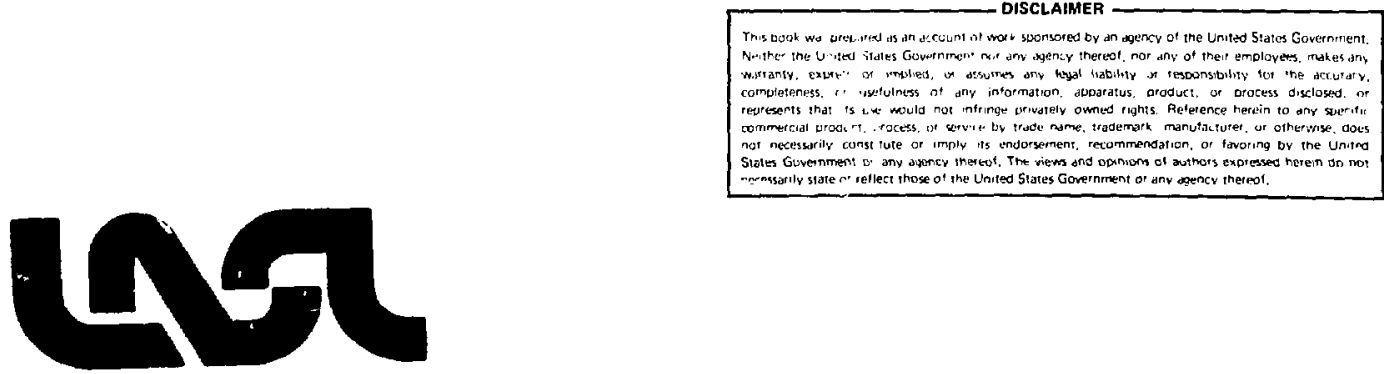

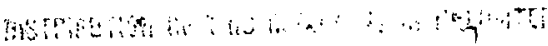


CONTENTS

ABSTRACT - - - - - - - - - - - - 1

I. INTRODUCTION $--\ldots-\cdots-\ldots$

II. BACKGROUND $--\cdots-\cdots-\cdots-\cdots$

III. CURRENT DEFINITION OF CLASS 8 AND CLASS 9 ACCIDENTS - - - - - - - - - - - 8

IV. LICENSING PRACTICE AND THE DEFINITION OF "ClASS 8" AND "ClASS 9" ACCIDENTS - - - - 10

v. COMPARISON OF "CLASS 8" AND "CLASS 9" ACCIDENT DEFINITION AND LICENSING PRACTICE AGAINST FINDINGS IN WASH-1400 - - - - - 17

VI. LICENSING TRENDS WITH RESPECT TO "CLASS 8" AND "ClASS 9" ACCIDENTS - $-\ldots 19$

VII. WORKABLE DEFINITIONS OF "ClASS 8" AND "ClAsS 9" ACCIDENTS - - - - - - - - - - 27

VIII. CONCLUSIONS AND RECOMMENDATIONS - - - - - 31 APPENDIX - - - - - - - - - - - 32 


\title{
LIGHT-WATER REACTOR ACCIDENT
}

\section{CLASSIF ICATION}

\section{by}

Beverly W. Washburn

\begin{abstract}
The evolution of existing classifications and definitions of 1 ight-water reactor accidents is considered. Licensing practice and licensing trends are examined with respect to terms of art such as "Class 8 " and "Class 9 accidents." Interim definitions, consistent with current licensing practice and the regulations, are proposed for these terms of art.
\end{abstract}




\section{INTRODUCTION}

This study has considered the matter of existing light-water reactor accident classifications, the potential need for defined classes of accidents, and possible classification schemes, with particular emphasis on the separation of so-called Class 8 and class 9 accidents by distinct boundaries. A review of the various accident classifications and their relationship to safety evaluation, environmental impact, and emergency planning was conducted. The increasing usage of phrases such as "Class 8 accident," a term of art without precise definition, is deemed to be a potential problem. A scheme of accident classification, believed to be consistent with existing regulations and licensing practice, is proposed.

\section{BACKGROUND}

The classirication of light-water reactor accidents has evolved over a period of time. Documentation relaied to safety analysis and evaluation has been reviewed to ascertain the extent to which the frequency of occurrence and the consequences or other criteria have been considered in the classification of accidents and to determine the role of postulated severe accidents in the safety evaluation process.

Part 100 of 10 CFR (27FR3509, April 12, 1962), which describes criteria for the evaluation of site suitability, indicates the expectation of an extremely low probability for accidents that could result in release of significant quantities of radioactive fission products. This regulation also requires an accident assumption for site evaluation purposes as follows. Calculations to aid in evaluating a proposed site should assume a fission product release based upon a major accident that would result in potential hazards not exceeded by those from any accident considered credible. Such accidents have generally been assumed to result in substantial meltdown of the core with subsequent release of appreciable quantities of fission products. 
Section 50.34 of 10 CFR Part 50 (33FR18612, December 17, 1968). which describes the technical information required in applications, requires an analysis and evaluation of the facility with the objective of assessing the risk to public health and safety resulting from operation of the facility. These analyses are to include a determination of (a) the margins of safety during normal operations and transient conditions anticipated during the life of the facility and (b) the adequacy of features provided for the prevention and the mitigation of the consequences of accidents. Appendix $A$ was added later to 10 CFR Part 50 to establish minimum requirements for the principal design criteria for facility features that permit the facility to be operated without undue risk to the health and safety of the public.

Appendix A of 10 CFR Part 50 (36FR3256, February 20, 1971), effectively created three classes of accidents by (a) defining anticipated operationa: occurrences in a quantitative manner, by expected frequency of occurrence and (b) introducing the concept of postulated accidents, a subset of which consists of loss-ofcoolant accidents, defined by initiating event (i.e., breaks of specified magnitude range in the reactor coolant pressure boundary). This creates two classes of accidents and leaves the possible third class, those accidents in neither class, unspecified. In event space, the relationship among the classes created by the regulations might be viewed as shown in Fig. 1. Anticipated operational occurrences are conditions that are foreseen or expected in advance and which are expected to occur one or more times during the life of the plant. Postulated accidents, the effects of which are used to evaluate design adequacy, are an assumed reality with no proof and hence logical means of assigning a meaningful expected frequency of their occurrence does not necessarily exist.

A proposed Annex to Appendix D, 10 CFR 50 (36FR22851, December 1. 1971), set forth standard required assumptions for nuclear power plant accidents to be used in the preparation of environmental impact statements. Appendix D of $10 \mathrm{CFR} 50$ has been superseded liy 10 CFR 51; however, Part 51 does not affect the status of the proposed Annex to Appendix D of 10 CFR 50 (39FR26279, 1974). The 


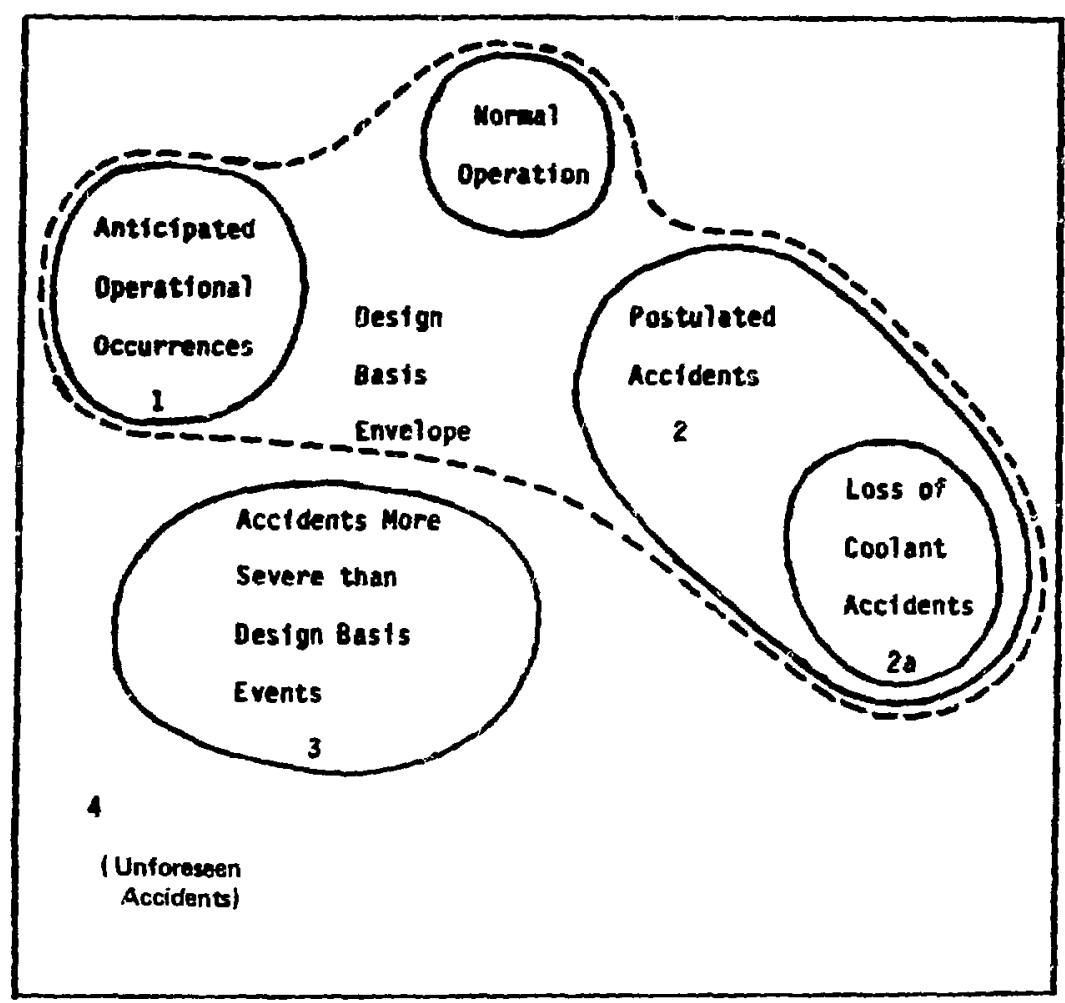

Fig. J. Event space.

proposed annex divided the spectrum of possible accidents, ranging in severity from trivial to very serious, into nine classes. It was presumed that each class can be characterized by an occurrence rate and a set of consequences. Representative types of accidents that must be analyzed in Environmental Reports were given for accident classes 1 through 8 . Of particular interest here are the definitions of class 8 and class 9 accidents. These two classes were defined as follows.

Class 8 - "Class 8 events are those considered in safety analysis reports and AEC staff safety evaluations. They are used ... as the design basis events to establish the performance requirements of engineered safety features." 
class 9 - "The occurrences in class 9 involve sequences of postulated successive failures more severe than those postulated for establishing the design basis for protective systems and engineered safety features. Their consequences could be severe."

Expected consequences and occurrence rates were not indicated. It is stated, however, that, although the consequences could be severe, the probability of occurrences in class 9 is so small (sufficiently remote) that their environmental risk is extremely low. With respect to Class 8 accidents, the Annex required that realistic evaluations, contrasted to the more conservative evaluations of the same events in Safety Analysis Reports, to be used for environmental risk.

Regulatory Guide 1.70, "Standard Format and Content of Safety Analysis Reports for Nuclear Power Plants," Revision 1 (October 1972), a revised document superseding a proposed guide issued in February 1972, recognizes that situations analyzed to accomplish evaluation of the safety of a nuclear power plant may range from anticipated operational occurrences to postulated accidents of low probability. In addition, the analyses should include an accident whose consequences are not exceeded by any other accident considered credible in order that the site evaluation required by $10 \mathrm{CFR}$ Part 100 may be conducted.

Chapter 7 of Regulatory Guide 4.2, "Preparation of Environmental Reports for Nuclear Power Stations," March 1973, contained essentially the same information that was in the proposed Annex to Appendix D, 10 CFR 50, above. Revision 2 of this Guide, July 1976, also contains the same classification as the proposed Annex. WASH-1250, "The Safety of Nuclear Power Reactors (Light ratercooled) and Related Facilities," July 1973, categorizes the range of accidents considered in assessing the risk to public health and safety from operation of the facility (10 CFR 50.34) into three classes as follows: 
1. Events of moderate frequency (anticipated operational occurrences) leading to no abnormal radioactive releases from the facility,

2. Events of small probability with the potential for small radioactive releases (abnormal radioactive release possible, but not expected) from the facility, and

3. Potentially severe accidents of extremely low probability, postulated to establish the performance requirements of engineered safety features and used in evaluating the acceptability of the facility site.

Representative types of accidents were given for each of the three classes. These examples differ in certain respects from those in the proposed Annex above: (a) Radwaste system accidents are given a lower frequency and possibly higher consequence than in the proposed Annex and (b) accidents identified in Classes 6 and 7 of the proposed Annex are believed to be representative of the third class above.

The draft of NUREG-0396, "A Modified Planning Basis for the Development of State and Local Government Radiological Emergency Response Plans in Support of Light Water Nuclear Power Plants," November 1977, sets forth four major groups of accidents and provides, in the glossary, a definition of class 9 accidents. This draft sicates: "The NRC staff has categorized postulated accidents into four major groups as follows:

"1. Events of moderate frequency (anticipated operational occurrences) leading to no significant radioactive releases from the facility.

"2. Events of low probability with the potential for small radioactive release from the facility.

"3. Very low probability but potentially severe accidents postulated to establish the performance requirements of engineered safeiy features and used in evaluating the acceptability of the reactor site. These events, some of which assume unlikely failures or fission product releases are referred to as design basis accidents (DBAs).

"4. There is a fourth group of accidents, the so-called 'Class 9' accidents, which includes any situation not 
specifically included in the groups of events above and typically is represented by some combination of failures which leads to core-melting and/or containment failure. These larger events are considered in the regulatory process by reducing their probability of occurrence to acceptably low values through design of the plant engineered safety features. The consequences of events in this group are not specifically analyzed in most applications. Such events, which are reduced to an acceptably low probability by design or siting requirements, include external hazards such as severe natural phenomena as well as accidents occurring within the facility."

Definition of the first three major groups is very similar, except for the choice of quantifying adjectives, to the categorization found in WASH-1250, above.

In addition, the glossary of NUKEG-0396 includes the following definition of a class 9 accident.

\begin{abstract}
"An accident considered to be so low in probability as not to require specific additional provisions in the design of a reactor facility. (Class 9 event sequences include those leading to total core melt and consequent degradation of the containment boundary and those leading to gross fuel clad failure or partial melt with independent failures of the containment boundary.)"
\end{abstract}

These definitions separate Class 8 and Class 9 events by a movable boundary, i.e., Class 9 contains all situations not included in the design basis (Class 8 ) and lower categories while the upper bound of situations considered in class 8 is not necessarily fixed. The phraseology of these definitions was changed slightly in the final report (December 1978). These changes are not regarded as substantial alteration of the definitions.

Additional classification and terminology is found in NUREG0438, "Plan for Research to Improve the Safety of Light-Water Nuclear Power Plants," April 12, 1978. The range of potential occurrences in a nuclear power plant was categorized into three major groups. 
1. Anticipated operational events, which may occur with moderate frequency (i.e., several times per year) and would not result in significant releases of radioactivity.

2. Events with a low probability of occurrence (in the range of 1 chance in 10 to 1 chance in 100 per year) and the potential for small releases of radioactivity.

3. Events with a very low probability of occurrence (in the range of 1 chance in 1,000 to 1 chance in 10,000 per year) for which engineered safety features are provided to mitigate potentially severe consequences.

By the definition in Appendix A, 10 CFR 50, both groups 1 and 2 above are anticipated operational occurrences. Group 3 is the postulated accidents, the effects of which are used to evaluate design adequacy. This categorization is similar, but not identical, to that found in WASH-1250, above. Although this categorization does not represent a new concept in accident classification, it does provide a quantization of event frequencies that had previously been stated only qualitatively. Although not within the scope of this study, it is of interest to apply these frequencies of occurrence and the accident consequences calculated in an environmental statement, for example, in a determination of the relative contributions of the various accidents and accident classes to risk. Low consequence, moderate to high frequency events may be greater contributors to risk than are high consequence, low frequency events.

III. CURRENT DEFINITION OF CLASS 8 AND CLASS 9 ACCIDENTS

The proposed Annex to Appendix D, 10 CFR 50, was published in 1971 with the expectation "that the provision of the proposed amendments (set forth in the Annex) will be useful as interim guidance until such time as the commission takes further action on them." (36FR22851). In 1974, the Commission revised other parts of its NEPA regulations, codifying them in $10 \mathrm{CFR}$ Part 51 , but left the proposed annex untouched. At this time, the Commission stated that the proposal was still under consideration, stressing that the new Part 51 does not affect the status of the proposed Annex (36FR26279, July 19, 1974). This document continues to be the 
Commission's interim guidance on the definition and treatment to be accorded accidents in environmental impact statements. It should be noted that this guidance was promulgated in the context of environmental impact considerations. It is presumed that strict interpretation would not result in its application to safety concerns.

Although not within the scope of this task, it seems appropriate to comment on the proposed Annex where the concept of nine "classes" of accidents apparently started. It is believed that the proposed Annex does not set forth defined classes or categories of accidents. The proposed Annex has created nine groups of situations, of which seven groups identify with specific location, system or operation in the plant where radioactivity may be released rather than with a consequence magnitude or other measure of severity. Situations, representative of each class and of those situations that must be analyzed, are given for eight of the groups. These situations do not appear to conform to a single principle in that they consist of initiating events, possible elements of event sequences, intermediate consequences of unspecified origin, or combinations of the foregoing. It is believed that this does not constitute good classification or definition.

The classification in the proposed Annex covers a spectrum of severity ranging from trivial to very serious. Although sets of probabilities and consequences were not assigned to each class in the proposed Annex, it is indicated that this is possible and required in the analyses. Review of typical environmental statements shows that the radiological consequences of postulated situations do not follow any uniform trend from trivial to serious between "Class I" and "Class 8." In some statements, the consequences of some postulated "Class 8 " events are comparable in magnitude to events associated with "Classes" 3 through 7 . If the probability of events in "Classes" 3 through 7 is comparable to or greater than that of the events in "Class 8" (as indicated in the earlier section on background), it follows that events in "Classes" 1 through 7 may make a contribution to overall risk comparable to or greater than that from "Class 8 " events. 
Appendix A, Appendix $K$, Section 50.34, and Section 50.46 of 10 CFR Part 50, establish the minimum requirements of the design basis envelope. It is believed that this envelope encompasses situations intended to be classified as "Class 8 " or lower in the proposed Annex. Other grouping or classification of events described in the foregoing background section are enlightening but they are not regarded as having the force of an understanding based on $10 \mathrm{CFR}$ Part 50 notwithstanding the status of the proposed Annex.

IV. LICENSING PRACTICE AND The DEFINItion OF "ClASS 8" AND "Class 9" ACCIDENTS

The actual events or situations that constitute so-called "Class $8, "$ in accordance with the current definitions and usage of that class, are those accidents that are or may be postulated for the purpose of evaluating the designs. Thus, licensing practice for design evaluation is always in accord with "Class 8 " even though such practice may expand or alter the scope of "class 8 " at a future time. Furthermore, "Class 9 " is currently defined in terms relative to "Class $8 . "$

In creating workable definitions of accident classes, it is necessary to consider current licensing criterla used for the evaluation and acceptance of plant designs. Appendix A of 10 CFR Part 50 (36FR3256, Fabruary 20, 1971) set forth the requirement to consider single failures in the design and evaluation of system features important to safety. In the design and evaluation of five features: residual heat removal, emergency core cooling, containment heat removal, containment atmosphere clean-up, and cooling water systems, it is required that, in addition to single failures, conditions of onsite power available with offsite power unavailable and vice-versa, be considered. The subsequent addition of Appendix K, 10 CFR Part 50 (39F'R1003, January 4, 1974) and Section 50.46 (39FR1002, January 4, 1974), additionally required consideration of the most serious concurrent single failure in the ECCS subsystem in carrying out the loss-of-coolant accident (LOCA) evaluation. Thus, in the evaluation of "Class 8" events, failure-of-function is not 
considered in features important to safety. However, many of these features may be considered, in the evaluation, to be operating with reduced, but adequate, performance because application of the single failure criterion in the analysis results in a partial loff-offunction in the as-designed system. The licensing evaluation of events, which are postulated to determine the acceptability of the designs, incorporates other conservative assumptions in the calculations or in component malfunctions or both.

Review of the USNRC Standard Review Plans indicates that the acceptance criteria for plant control and accommodation of postulated transients and accidents may be categorized, according to consequences, into four general categories. This review also observed that a qualitative specification of event frequency was associated with some, but not all, events covered by these review plans. Using the information in the standard Review Plans, these events can be grouped according to the following categorization of the acceptance criteria used for the safety evaluation:

1. events of no consequence having unspecified frequency of occurrence,

2. everts of small consequence having moderate frequency of occurrence,

3. events of moderate consequence occurring infrequently, and

4. events of potertially severe consequence having unspecified frequency of occurrence [the probability of events in this category has been previously described, for example, as low (Regulatory Guide 1.70), very low (NUREG-0396 and NUREG-0438), and extremely low (WASH1250 and Section 100.10 of 10 CFR Part 100)]. Design basis LOCAs and "Class 8 " events are in this category.

The safety evaluation acceptance criteria associated with the above categorization is as follows.

\section{Category 2 -}

a. Pressure in the reactor coolant and main steam systems should be maintained below $110 \%$ of the design pressures. 
b. Fuel clad integrity should be maintained by ensuring that the following criterion is satisfied throughout the transient. General Design Criterion 10 requires that the reactor core be designed with appropriate margin to assure that specified acceptable fuel design limits are not exceeded during any condition of normal operation, including the effects of anticipated operational occurrences. There are two acceptable approaches to meet this criterion:

(1) For departure from nucleate boiling ratio (DNBR) or critical heat flux ratio (CHFR) correlations there should be a $95 \%$ probability, at the $95 \%$ confidence level, that no fuel rod in the core experiences a departure from nucleate boiling condition during normal operation or transients that are anticipated to occur with moderate frequency.

(2) For critical power ratio (CPR) correlations, the limiting (minimum) value of CPR is to be established such that $99.9 \%$ of the fuel rods in the core would not be expected to experience boiling transition during core-wide transients. For transients that effect only a portion of core, the same value of CRP is used to provide additional conservatism.

c. An incident of moderate frequency should not generate a more serious plant condition without other faults occurring independently.

d. An incident of moderate frequency in combination with any single active component failure, or single operator error, should not cause loss-of-function of any barrier other than the fuel cladding. A limited number of fuel rod cladding perforations is acceptable.

Category 3 -

a. For infrequent incidents, the plant should be designed to limit the release of radioactive material to assure that doses to persons offsite are kept to values which are a small fraction of 10 CFR Part 100 guidelines. b. The event should be accommodated with the failure cif only a small fraction of the fuel rods in the reactor, 
and the core geometry should remain intact so there is no loss of core cooling capability. Safety functions should be accomplished assuming the worst single failure of a safety system active component.

c. The event should not, by itself, generate a more serious condition or result in a loss-of-function of the reactor coolant system or containment barriers.

d. Pressures in the reactor coolant and main steam systems should be maintained below 1108 of the design pressures.

\section{Category 4 -}

(These events are design basis loss-of-coolant accidents resulting from a spectrum of postulated pipe breaks within the reactor coolant pressure boundary. "Class 8 " events are in this category.)

a. The calculated maximum fuel rod cladding temperature does not exceed $2200^{\circ} \mathrm{F}$.

b. The calculated maximum local oxidation of the cladding does not exceed $17 \%$ of the total cladding thickness before oxidation.

c. The calculated total amount of hydrogen generated from the chemical reaction of the cladding with water or steam does not exceed 18 of the hypothetical amount that would be generated if all of the metal in the cladding cylinders surrounding the fuel, excluding the cladding surrounding the plenum volume, were to react.

d. Calculated changes in core geometry are such that the core remains amenable to cooling.

e. After any calculated successful initial operation of the ECCS, the calculated core temperature is maintained at an acceptably low value and decay heat is removed for the extended period of time required by the long-lived radioactivity.

f. The total potential consequences at the nearest exclusion area boundary and at the low population zone outer boundary are well within the guidelines of 10 CFR Part 100. 
The foregoing information appears to present a consistent licensing practice with respect to "Clase 8 " and lower events.

Licensing practice with respect to "Class 9" events has been similar for both the safety and environmental reviews. While recognizing that the consequences of these events could be severe, the Staff (Staff Memorandum, September 1, 1971) and the Commission (the proposed Annex, December 1, 1971), believe that there exists a high degree of assurance that these potential events are sufficiently remote in probability to make their environmental risk extremely low. The philosophy underlying the Commission's position that consequences of "Class 9" events need neither be considered nor subjected to ameliorative design features is based on their improbability. This understanding and the position of the Commission on not considering these events in the safety and environmental reviews have been firmly established and confirmed by a long line of Appeal Board and judicial decisions in the face of vigorous challenges. It is believed, however, that "class 9 " events are considered by licensing when a mechanism for causing them can be shown. The ATWS issue, for example, is believed to involve this type of "Class 9 " event. This issue has been under consideration by licensing for several years and final resolution is pending. Although the issue is not resolved, required modifications have been implemented in some BWRs as short-term fixes for ATWS. Other aspects of the ATWS concern will be discussed in the following sections of this report.

Environmental reviews have been generally limited to accidents in "Class 8" and lower using realistic analysis assumptions. Recently, however, the NRC staff included consideration of "Class 9 accidents" in the Floating Nuclear Plant (FNP) Environmental Impact Statement and it is of interest to briefly trace the history of this action. At the behest of the ACRS, the NRC staff undertook a liquid pathway generic study to compare the consequences of accidental releases of radioactivity into the ocean surrounding floating nuclear plants with consequences of such releases at land-based reactors. This study was prompted by the offshore Power systems (OPS) application but was carried out as a generic analysis. 
The staff chose to consider consequences of "Class 9 accidents" in the study and, in a departure from usual practice, in the offshore Power systems (OPS) licensing proceeding as well. A draft report, "Liquid Pathway Generic Study," (LPGS) NUREG-0140, was issued in september 1976. ACRS and outside criticisms of the LPGS necessitated revision of the draft and the final IPGS report (NUREG0440) was published in February 1978.

A Generic Environmental Statement considering the siting and operation of Floating Nuclear Power Plants, Part II of the Draft Environmental statement (DES) related to manufacture of Floating Nuclear Power Plants (STN 50-437), NUREG-75/113, was issued in November 1975. At this time, it was stated that this DES would be supplemented at a later date with a generalized study which compares the consequences of FNP accidents with those of land-based plants for a spectrum of releases. This supplement was to become a third separately prepared element (Part III) of the environmental review of the OPS application for manufacturing FNPs. Part II of the Final Environmental statement (FES) was published in september 1976. Part III of the FES was published in draft form in October 1976. However, criticism of the LPGS report necessitated revision of this draft. A revised draft of the FES Part III was produced in May 1.978 and the FES, Part III, was published in December 1978. OPS moved the Licensing Board to order the staff to exclude assessment of the "Class 9 accident" from the FES. The board denied the OPS motion on February 23, 1978 and on March 30 declined to reconsider that denial. OPS asked the board to certify the question of the need to discuss the "Class 9 " question. In response, the board ruled ( $A L A B-489$, August 21, 1978) that the consequences of "Class 9 accidents" may be considered in this environmental statement. This ruling carried no connotation that the staff's judgments expressed in the FEs are necessarily sound, much less that its recommended license conditions are warranted. These matters remain yet to be explored in the pending proceedings before the Licensing Board.

As a consequence of considering "Class 9 accidents" in the OPS application, the staff recommended that the planned concrete 
base-mat in the containment building be replaced with one made of refractory material (MgO).

The question of the legality of the NRC Staff's considering "Class 9 accidents" has been raised to the NRC and the Commission has agreed to hear the matter. Briefs were filed January 12, 1979, and at the time of this writing, the Commission has reportedly ruled in favor of the NRC Staff.

The Atlantic Generating Station DES (NUREG-0058) related to construction was issued April 1976. Environmental consideration was limited to "Class 8 " and lower accidents. This DES was revised October 1976 to include consideration of the accidental liquid pathway releases developed in the draft of the LPGS (NUREG-0140). At this time, the staff concluded the following.

- In general, it appears that any event considered within the design basis envelope is not likely to lead to a significant radioactivity release to the environment via the liquid pathway. However, should such a release occur, the consequences are expected to be small.

- For a given initiating event, the likelihood of a release to the liquid pathway is significantly less than to the gaseous pathway.

- The floating nuclear plant, by virtue of close proximity to water, would seem to have the potential for significantly different consequences than land-based plants in the event of very severe accidents involving coremelt. However, review of the events leading to coremelt and ultimate containment penetration indicate that the expected consequences are generally comparable.

The NRC staff stated that more in-depth assessments would be required to further refine the results of this study and that in any event, it does appear that consequences of accidental releases for a FNP at the AGS site can be made low, comparable to land-based sites. 
Revision of the LPGS report necessitated revision of these findings also.

v. COMPARISON OF "ClASS 8" AND "ClASS 9" ACCIDENT DEFINITION AND LICENSING PRACTICE AGAINST FINDINGS IN WASH-1400

The Reactor Safety Study (WASH-1400) considered mainly accidents in which safeguards fail to provide the level of protection necessary to prevent core melt. These accident sequences include those that are the major contributors to risk. These sequences were included in WASH-1400 release categories PWR-1 through -7 for the PWR and release categories BWR-1 through -4 for the BWR.

Some accident sequences that do not result in core melt, PWR release categories 8 and 9 , and BWR release category 5 , were also considered. For the PWR analyses, these sequences considered the LOCA accompanied by failure of $\mathrm{NaOH}$ addition to the containment spray, failure of containment spray injection, or failure to adequately isolate containment openings and penetrations. Release category PWR-8 approximates a PWR design basis accicient (large pipe break), except that the containment would fail to j.solate properly on demand. The other engineered safeguards are assumed to function properly. Release category PWR-9 also approximates a PWR design basis accident (large pipe break) in which the release of the radioactive iodines and alkali metals into the containment would be a very small fraction of the release assumed in release category PWR-8. The minimum required engineered safeguards would function satisfactorily to remove heat from the core containment. Consequences in release categories $\mathrm{PWR}-8$ and -9 are regarded as minor.

BWR accident sequences that do not result in core-melt were considered in release category BWR-5. This category approximates a BWR design basis accident (large pipe break) in which it is assumed that the minimum required engineered safeguards would function satisfactorily.

As defined in the proposed Annex, "Class 8 " events are those considered in licensing safety evaluations as design-basis events to establish the performance requirements of engineered safety 
features. Radioactivity releases from these events would be comparable to those associated with WASH-1400 release categories PWR-8 and -9 for the PWR and with release category BWR-5 for the BWR. One design basis accident, used in connection with site suitability evaluations to establish compliance with 10 CFR Part 100, is a LOCA with an assumed large fission product release from containment. Releases to the containment assumed in the licensing review and evaluation of this event are comparable to those associated with a WASH-1400 core-melt release category between PWR-7 and PWR-8 or between BWR-4 and BWR-5. The release pathway and assumed release rates to the environment, however, differ from those for PWR- 6 and PWR-7 releases in WASH-1400. These differences are believed to result in the radiological consequences of the site suitability evaluation accident possibly exceeding those associated with release categories PWR-8 or -9 , dependent on the filter performance. For the BWR, the site suitability evaluation accident release to the environment would correspond to a release between categories BWR-4 and BWR-5. Other events, such as rod ejection (PWR), rod drop (BWR) and steam line breaks, defined as "Class 8" events in the proposed Annex were not analyzed in the RSS. Radiological consequences of "Class 8 " accidents included in the environmental statements for 11 nuclear power plants were used to estimate the RSS release categories for these accidents that were not analyzed in the RSS. The PWR rod ejection accident, with adequate safeguards performance, is comparable to a small LOCA and would have a release associated with RSS release category PWR-9 or higher, if such category existed. The PWR steam line break accident would have a release associated with a RSS release category higher than PWR-9, if such category existed. The BWR rod drop accident and the steam line break accident, with adequate safeguards performance, would have releases associated with RSS release category BWR-5 or higher, if such category existed.

From these considerations, it is believed that "class 8 " events, as defined in the proposed Annex and as considered in the safety evaluation in the licensing process, would be associated with RSS release categories PWR-8 or higher and BWR-5 or higher. It also 
follows that "Class 9" events, as defined in the proposed Annex, would be associated with RSS release categories PWR-7 and lower and BWR-4 and lower. The majority of the accident sequences considered in the RSS are "Class 9 " events involving sequences or combinations of successive failures more severe than those postulated for establishing the design basis for protective systems and engineered safety features and for site evaluation purposes. These RSS sequences involve the failure of one or more safeguard features to provide adequate performance.

The ATWS concern was mentioned in the previous section. Review of the analysis in WASH-1400 shows that the ATWS event in a BWR is a dominant contribution to the overall BWR risk and has the potential to result in core-melt without being accompanied by additional or successive failures. The potential for ATWS in the BWR to lead directly to core melt, as analyzed in WASH-1400, makes this an important safety concern. ATWS in the PWR is not a significart contributor to overall PWR risk and it must be accompanied by successive failures (such as failure of the primary system safety and relief valves to provide adequate primary relief capacity or failure of these valves to reclose after opening) in order to result in core melt. On this basis, ATWS in the BWR is regarded as a "Class 9" event while in the PWR it may be "Class 9" (in terms of the number of failures required to produce it) or lower (in terms of its consequences) depending on the assumed accompanying failures.

VI. LICENSING TRENDS WITH RESPECT TO "ClASS 8" AND "CLASS 9" ACCIDENTS

Resolution of generic issues, Regulatory Staff and ACRS concerns and the results of regulatory safety research projects have potential impact on licensing practice related to "class 9 " accidents.

It is believed that licensing practice and trends with respect to accidents can be placed into better perspective if issues, concerns, and topics addressed in licensing proceedings and those under consideration by the NRC can be assigned to an accident class 
and additionally classified as to whether they are accident prevention measures or accident consequence mitigation measures. A large portion of the licensing effort is directed at the prevention and mitigation of consequences at the "Class 8 " level. It is believed that features incorporated to prevent and mitigate accidents in "Class 8" are preventative measures against many "Class 9" accident scenarios and that some of these features may be properly recognized as having a degree of effectiveness in mitigating the effects of "Class 9 accidents." However, it is believed that plant designs and safeguards have not specifically addressed the core-melt scenarios in "class 9 " because such sequences fall beyond the design-basis envelope.

The Congress has placed some impetus on the resolution of unresolved safety issues. Section 21D, Energy Reorganization Act of 1974, as amended (PL 95-209), requires reports on the progress of the NRC program for the resolution of unresolved safety issues and the progress on individual tasks therein in the Commission's Annual Report to Congress, beginning with the 1978 Annual Report. The NRC Report to Congress, January 1, 1978 (NUREG-0410), described the plans and program. Also included in this report were the identification and description of safety issues that were unresolved on the date of enactment of the legislation. The ACRS identified 30 items in a list limited to generic items cited specifically in its letters pertaining to projects and related matters. The NRC Staff identified 133 items classified by relative importance to safety and priority for resolution. Due to time limitations, the scope and intent of all these issues have not been reviewed in detail. However, most of these issues are regarded as falling within the scope of the design-basis envelope. That is, they are preventative or mitigative measures for "Class 8 " or lower accidents, they relate to the prevention of the progression of "Class 8 " situations into "Class 9 accidents," or they are issues that could lead to improved understanding of technical detail or to refinements and improvements in the licensing process. Some of these issues could, however, have significant impact on the reactor licensing process. 
Three issues under consideration, ATWS, common mode failures (particularly in the scram and power systems), and primary system overpressure at low temperatures, are believed to be "Class 9" events that are potentially capable of resulting in core-melt without additional failures. Although these are judged to be only a small contribution to the core-melt probability, their potential for leading directly to severe consequences outside the designbasis envelope warrants their resolution.

Although the ATWS issue is not resolved, its eventual resolution has the potential for influencing licensing trends with respect to "Class 8 " and "Class 9" accidents. If the issue is resolved and the probability and the consequences of ATWS can be established, the value of these parameters could become guideline values for licensing safety objectives. In WASH-1270, a report on ATWS (September 1973), the Regulatory Staff stated:

\begin{abstract}
"The safety objective is that the likelihood of all accidents with significant consequences not included in the design-basis envelope should not be greater than one chance in one million per year, i.e., should not occur with a failure rate greater than $10^{-6}$ per year. For the particular potential failure path of ATWS, the staff believes that a failure rate of the order of one-tenth of the overall safety objective is an appropriate objective"
\end{abstract}

(i.e., a rate of $10^{-7}$ per year). This phraseology is suggestive of requiring the total likelihood of all accidents having consequences greater than those included in the design-basis envelope (one of which is ATWS) to be less than one chance in one million per year with one-tenth of the aggregate objective being allowed to the ATWS event. Two recent statements appearing in NRC documents should also be noted because of their potential as safety guidelines and because it is believed that their basis is a possible departure from the minimum acceptance criteria based upon Title 10 , Code of Federal Regulations. With particular reference to ATWS in 
NUREG-0460, April 1978, the following statement is made by the Staff :

\author{
"We recommend that a safety objective of $10^{-6}$ unac- \\ ceptable ATWS events per reactor-year is more \\ appropriate"
}

(than the expected frequency of $2 \times 10^{-4}$ per reactor-year),

\begin{abstract}
"and therefore, that some corrective measures to reduce the probability or consequences of ATWS are required."
\end{abstract}

A similar stated objective appears in NUREG-0328, Vo1. 4, Number 6, December 16, 1978, Status Summary Report (Pink Book) as follows:

"The staff safety objective is that the likelihood for accidents with significant safety consequences not inciuded in the design-basis envelope should not be greater than approximately $10^{-7}$ per reactor-year."

Although this statement appears in connection with the ATWS review summary, the wording is suggestive of broader application.*

The NRC staff has reported (draft of the Annual Report, SECY78-616A, December 28, 1978) progress on 17 issues involving 22 tasiss in the NRR Program for the Resolution of Generic Issues. The issue of reactor vessel transient overpressures has been resolved by implementing procedural controls for PWR facilities, which reduced the frequency of occurrence, and by implementing equipment changes, which restrict transient pressures to acceptable

*The NRC staff now believes that a numerical safety objective is not satisfactory for use in regulatory decisionmaking as previously suggested (NUREG-0460, Vol. III, page 3). 
levels. This leaves common mode failures in electrical power systems and ATWS as issues whose resolution may impact licensing consideration of "Class 9 accidents."

Parts of NRC's ongoing safety research program, studies of new siting concepts, improved offsite emergency response planning and, improved reactor shutdown systems, are directed at "Class 9 accidents." NRC's proposed plan for research to improve the safety of LWR plants incorporates additional research topics that are believed to be likewise directed at "Class 9 accidents." Topics being proposed in the "Class 9 " area are alternate containment concepts and alternate emergency core cooling concepts. Under consideration for safety research are reactor.vessel rupture control and core retention measures.

In terms of the number of topics, approximately $50 \%$ of the NRC research for improved reactor safety is directed at "Class 9 accidents" with the topics divided about equally between prevention and mitigation. With the exception of reactor vessel rupture control and core retention measures, these topics involve the extension and improvenent of features, which are currently considered within the design-basis envelope, to a performance level that will reduce the consequences or expected frequency of "class 9 accidents." Consideration of any of the foregoing safety research topics in the licensing process would result in extension of the design-basis envelope to cover core-melt ("Class 9") accidents.

The extensive consideration of "class 9 " events and related risks in the offshore Power Systems application for a license to manufacture Floating Nuclear Power Plants (FNP) is a significant departure from past practice and a possible element of a licensing trend. Further pursuit of core-melt related concerns in this application is, in part, contingent on the NRC Commissioners' decision regarding the legality of the NRC staff's actions.

In the environmental review of this application (FES, Part III, NUREG-0502, December 1978), the NRC staff concluded for postulated design-basis accidents in FNPs: 
- The risk of airborne radioactive releases is low. [Equivalent to that of a land-based plant (LBP)].

- The risk of radioactive releases to the liquid pathway are very low (as is that for an LBP) and not significant compared to the risk for airborne releases for the same accident.

- The overall risk of radioactive releases to the environment is low (comparable to that for an LBP).

Presentation in the FES, Part III, of NRC staff requirements for FNP plant design and site modifications addressing core-melt accidents was a significant departure from the original intent that this part of the FES was to present the results of a generalized study comparing the consequences of FNP accidents with those of land-based plants for a spectrum of releases (see DES, Part II, NUREG-75/113, November 1975). Also, since the detailed consideration of "Class 9 " events came into being in connection with this application, subsequent to the FES, Part II, September 1976 (which considered "Class 9 " and found the risk to be extremely low) and revision of the LPGS, it might be believed that the liquid pathway considerations are in some way involved in the current NRC requirement for FNP plant design and site modifications. The following information (FES, Part III, December 1978) does not support such belief.

For postulated core-melt accidents, the NRC staff concluded:

- The overall risk at an ocean-sited FNP falls near the upper portion of the range of risk for existing LBP designs. The risk associated with the ocean-sited FNP, as presently designed, may not compare as well with recent and future LBP designs.

- The overall risk of releases to the environment is greater for an FNP sited in an estuary than for the same plant sited offshore in the ocean.

- Much smaller airborne release would be expected for about half of the current LBPs. (This statement does not really state the airborne risk of an FNP, which this ES shouid be addressing.) 
The staff did not give the risk of airborne and liquid pathway releases for the postulated core-melt accidents (as they did for design basis events). Instead, they developed a monetized risk to permit comparison of these accidents between FNPS and $t_{\lrcorner B P S}$ and concluded:

- The monetized risk of airborne releases is low (comparable to that of the LBP),

- The monetized risk of liquid pathway releases for the most likely case is low (generally greater than those of the LBP), and

- The monetized overall risk, exclusive of ecological costs, is relatively low for an estuarine sited FNP.

Evidently, the design basis accident risks from the FNP are sufficiently comparable to those from an LBP to make the proposed FNPs acceptable, from the standpoint of environmental impact, for licensing. Extension of the FNP considerations to "Class 9," coremelt accidents, resulted in an overall FNP risk that was "near the upper range" of that for "existing LBP designs." [The concept of recent, existing, future, etc., LBP designs has been introduced into the licensing considerations of FNP risk and extensive discussion of qualitative perceptions of the LBP relative risk from current designs, existing designs, recent designs, future designs, current generation, operating reactors, and proposed reactors (actual terms used in the ES) is found in the ES.]

Review of prior NRC environmental statements relating to what is believed to be "existing LBP designs" shows chat the NRC staff believes that these designs provide and maintain a high degree of assurance that potential accidents in "Class 9" are, and will remain, sufficiently small in probability that the environmental risk is extremely low. Evidently the marginal difference in very qualitative environmental impact findings for existing LBPs and the FNP (i.e., "near the upper range" of an "extremely low risk") is taken as justification to require plant design and site modifications to accommodate the "Class 9" accidents in order to demonstrate acceptability of the design for licensing. After the applicant took 
exception to the NRC Staff's request that these modifications be made, the staff took upon themselers the investigation of plant design modifications to accommodate core-melt accidents. They considered characteristics of sacrificial basemat materials and available materials, estimated the melt-through time for a sacrificial bed of MgO, sought expert opinion on the use of sacrificial materials to delay melt-through in the event of a core meltdown accident in an FNP, and "concluded that there is reasonable assurance that a material can be selected that will perform satisfactorily to substantially increase the melt-through time and reduce the airborne release by reduced gas generation in the melt" (Section E.6). The underlying rationale for reducing the generation of noncondensible gases, produced in the decomposition of concrete during the melt-through process, appears to be that the ice condenser containment concept, having low containment design pressure and relatively small volume, has been shown to exhibit containment failure by overpressure from these gases prior to failure by basemat melt-through (Sec. 3.4.1.1). (Overpressure has not been verified as the primary failure mode of the containment. There is some evidence that this is not the primary failure mode of the proposed design.) This concern is believed to apply also to 12 known LBP ice condenser design's; one in operation and 11 in licensing review. The necessity for substantially increasing the meltthrough time has not been demonstrated. The FES discussed a spectrum of countermeasures made possible by the increase in meltthrough time. Most of these involve the tacit assumptions that personnel will be available and that they will accept the risk associated with implementation. (Although not clearly stated in the FES, PART III, it might be inferred from the discussion in this document that the need to increase the melt-through time is the justification for the required design modification in the basemat. This interpretation is supported by a statement made by Harold Denton, NRC, at the ACRS meeting, February 8, 1979, indicating that the need for implementation of countermeasures led to the decision to require these modifications.) In Part III of the FES, the NRC Staff also considered a number of concepts in administrative 
controls and additional design features specifically related to coremelt accidents. A few of these concepts might reduce the likelihood of a core-melt accident but most are directed at mitigation of the consequences of these accidents. "Class 9" event licensing actions subsequent to the completion of this report are summarized in the Appendix .

VII. WORKABLE DEFINITIONS OF "CLASS 8" AND "ClASS 9" ACCIDENTS

Although it is believed that nine classes of accidents may not exist, workable definitions of "Class 8 " and "Class 9" accidents may be based on:

1. levels of system failures in possible accident sequences, specified with or without specific consideration of consequences,

2. guideline values of consequences, or

3. guideline values of risk, either relative or absolute.

The foregoing background review indicates that the licensing trend, with respect to accident classification; has been toward using risk, albeit qualitative, as a classification basis while past licensing safety evaluation practice, using failure modes and effects analyses, is more closely associated with accident definitions based on levels of system failures and specific effects. The failure modes considered in these analyses are established by the criteria and requirements of 10 CFR Part 50 while the detailed acceptance criteria for the effects have been evolved by the licensing staff albeit that guideline values of consequences are established by $10 \mathrm{CFR}$ Part 100. Although consequences of accident sequences reprasent an incomplete understanding of the hazard involved, a consequence criterion is in keeping with that portion of current licensing practice where acceptability of the site and system design is determined by dose guidelines set forth in 10 CFR Part 100.

While lacking completeness, considerations based on accident consequences avoid introduction of the issues concerning the assessment of the very small probabilities of occurrence expceted 
for "Class 8" and "Class 9" events. The dose guidelines set forth in $10 \mathrm{CFR}$ Part 100 could, for example, form a convenient consequence boundary between "Class 8 " and "Class 9" accidents. These specific guideline values are not, however, appropriate for this boundary because the consequences of many possible "class 9 " (core melt) accident sequences, as currently defined, do not exceed these guideline values of offsite exposures (Draft NUREG0396, November 1977). A boundary established by the 10 CFR 100 guideline consequence values would have the effect of arbitrarily expanding "Class 8 " to encompass events having consequences more severe than those presently considered in licensing safety evaluations. Thus, this boundary is not consistent with current licensing practice.

Other guideline values of consequences, in particular, a value to be used to distinguish between "Class 8 " and "Class 9" events, might be developed for the purpose of accident classification. However, there is concern over the potential misuse of consequences, whether their magnitudes are small or large, and over the possible effects of the conservatisms used in the modeling, particularly as they may reflect nonuniformly in the calculated consequences or classification of a spectrum of accident sequences.

While these observations would not seem to preclude guideline values of consequences to provide a workable defined distinction between "Class 8 " and "Class 9" accidents, it is believed that general classification on the basis of consequence is not a preferred approach.

While none of the bases for accident classification are free of problems, a classification based on some form of risk is believed to be preferred because risk encompasses the notions of both the consequence and the frequency of occurrence of the accident and provides more complete understanding of the accident.

The importance or significance of an accident cannot be determined by either of these qualities taken separately. While a method of classification based on risk is believed to be preferred over other schemes, present circumstances appear to discourage using risk. Our current knowledge of risk from accidents in 
Classes 1 through 8 is not sufficiently complete to permit the assessments necessary in formulating such classification.

There are aiso two concerns of a more serious nature that apply to the likelihood of the events in "Class 8 " and "Class 9." First, in analyzing the probability of a future event that has never occurred in the past, the confidence in the correctness of the result becomes an issue. Secondly, there is a lack of confidence in the absolute assessment of the very smali probabilities expected for "Class 8 " and "Class 9" events. This is particularly true when such probabilities are dominated by relatively few elements in the chain of events. If accidents are to be classified according to risk, other considerations also need to be addressed. For example, the risk parameter chosen for quantification of the accident classes may involve site specific factors. In this case the dependence of risk on these factors could result in siting, rather than plant design, being a dominant factor in determining the class of a postulated accident. Risk parameters that do not involve site specific factors could be chosen for accident classification. However, consideration of the plant without regard for its siting represents incomplete understanding of the importance of postulated accidents.

Interim definitions of "Class 8 " and "Class 9 " accidents are appropriate until more complete definitions can be reliably related to risk. Definitions in accord with existing licensing regulations and practice will be proposed.

Section 50.34, Sec. 50.46, Appendix A, and Appendix $K$ of 10 CFR Part 50, set forth the designs and systems that must be included in the LWR plant and that must perform their intended functions in the presence of the postulated failures specified by these regulations. These regulations define those possible paths in accident event sequences that involve degraded system function and for which the outcomes must be acceptable in termis of public health and safety. The postulated failures required to be considered in the designs and in the safety evaluations may result in degraded system performance, but not the failure of function, of engineered safety features. These degraded performance accident 
sequences define the design-basis envelope and they are "Class 8" accidents, as currently defined. The distinction between degraded performance and failure of function will be used to establish interim definitions of "Class 8 " and "Class $9 "$ accidents and to provide demarcation of these two classes.

The proposed interim definition of "Class 8 " accidents:

Those accident sequences in which the engineered safety features pertorm their intended function in the presence of postulated failures that are required, by the licensing regulations, to be considered in the designs and safety evaluations.

It is believed that this definition is in accord with current licensing practice and the proposed Annex.

Two interim definitions of "Class 9" accidents are proposed for consideration.

1. Those accident sequences in which the engineered safety features fail to perform their intended function in the presence of postulated failures that are in excess of those required, by the licensing regulations, to be considered in the designs and safety evaluations.

Or an alternate definition consisting of two parts:

2. a. A LOCA accompanied by the failure of function, including partial operation not alequate to provide the required function, of at least one of the following: (1) electric power or (2) core residual heat removal, or (3) emergency core cooling, or (4) containment heat removal (PWR), or (5) containment atmosphere clean up (PWR), or (6) cooling water system. (Core meltdown following LOCA is assumed to be the result of failure of any of these features.)

b. A transient requiring reactor shutdown from hot operating conditions accompanied by any of the following: (1) loss of electric power and loss of decay heat removal (PWR), or (2) failure to scram and failure of reactor coolant system safety or relief valve to reclose (PWR), or (3) failure to remove decay heat (BWR), or (4) failure to make reactor subcritical (BWR). 
It is believed that these definitions of "Class 8 " and "Class 9 " accidents distinguish the two classes by the number of failures considered in each class and by performance vs nonperformance of the intended system function.

\section{CONCLUSIONS AND RECOMMENDATIONS}

This review shows that nine distinct classes of accidents probably do not exist and that so-called "Class 8" and "Class 9" accidents may have a better defined separation, due to actual licensing practice, than their existing definitions tend to indicate. The categorization of postulated accidents into four major groups in NUREG-0396 represents the best overall classification to date. This classification would be enhanced if the catchall phraseology defining the fourth group of accidents (so-called "Class 9") were to be eliminated and if the concepts included in each group were more precise.

Workable interim definitions of "Class 8" and "Class 9" accidents, based on the number of failures and system performance level, were proposed in Sec. VII.

In the FES, Part III, related to Floating Nuclear Power Plants, the NRC staff went considerably beyond the intended scope of comparison of overall risk from accidental releases for floating and land-based nuclear power plants. The major portion of the consideration was directed toward core-melt accidents and comparison of their impacts. In addition to this, however, the NRC staff considered means for mitigating the consequences of core-melt accidents in the floating nuclear plant and in land-based plants. In this application, the NRC staff requires specific core-melt accident consequence mitigation features in the plant design. Review of the rationale for these requirements indicates that they are not based on system concepts or design features that are unique to the FNP. Therefore, it is believed that these requirements may be applied to LBPs also. A general lack of coherence and the introduction of factors that were extraneous, though somehow related, in the core-melt accident considerations preclude 
detailed understanding of the issues and create a concern for the soundness of the judgments expressed and for the ultimate impact of this FES on the licensing process.

\section{APPENDIX}

The accident at Three Mile Island (TMI) and licensing actions on "Class 9" events have occurred since this report was completed. These ongoing licensing actions will be summarized in this appendix.

As a consequence of the Offshore Power Systems (Floating Nuclear Nuclear Power Plants) appeal of the consideration of "Class 9 " accidents, in september 1979, the NRC directed the staff, before a formal rulemaking proceeding on this subject, to:

1. provide recommendations on how the guidance of the Annex might be modified on an interim basis pending completion of the rulemaking to reflect recent developments and current staff policy and

2. bring to the Commission's attention any individual cases in which the staff believes the environmental consequences of "Class 9" accidents should be considered.

The Susquehanna licensing board memorandum and order conderning Class 9 Accident Contention, October 19, 1979, Susquehanna Steam Electric Station, Units 1 and 2, LBP-79-29, concluded that the occurrence of the accident at TMI-2 constitutes a prima facie showing as to the probability of occurrence of that specific acs.dent sufficient at least to form the basis for an admissible contention.

In a preliminary response of the staff to the Commission's offshore Power Systems decision, the staff has stated its intention 
to develop for Commission consideration, by January 1980, a policy statement which as an interim measure would withdraw the old proposed Annex and abandon the system of classes of accidents in favor of a continuum representation of the probability of exceeding selected consequences based upon developments in quantitative risk assessment techniques and in the light of the TMI-2 accident ("Class 9 Accident Considersions," SECY-79-594, October $31,1979)$.

In the suspension of $10 \mathrm{CFR} 2.764$ and Statement of Policy on Conduct of Adjudicatory Proceedings (44 Fed. Reg. 65049, November 9, 1979), the Commission stated the following.

In reaching their decisions, the Boards should interpret existing regulation and regulatory policies with due consideration to the implications for those regulations and policies of the Three Mile Island accident. In this regard, it should be understood that as a result of analyses still under way, the Commission may change its present regulations and regulatory polisies in important respects and thus compliance with existing regulations may turn out to no longer warrant approval of a license application.

In a recent decision of the Appeal Board, Black Fox Units 1 and 2, ALAB-573, December 7, 1979, that board pointed out that the Commission is rethinking the policy, formulated in the proposed Annex, against considering "Class 9" accidents. The ASLB hearing the matter of the restart of TMI, Unit 1 , ruled that contentions which use the actual events at TMI as a base and then add or change a credible specific occurrence or circumstance, set forth sufficiently specific accidents which have a close nexus to the TMI accident and such contentions are, therefore, admissible. This board also held, on the basis of the foregoing Commission statement, that no further special showing is required of intervenors to admit a contention alleging a specific "Class $9 "$ accident which is either the same as or closely related to the actual accident which took place at TMI-2 (ASLB First special Prehearing Conference Order, December 18, 1979). This board also directed the staff to inform the board and the commission whether 
or not any specific accident sequence, which has a reasonable nexus to the TMI-2 accident and which heretofore may have been regarded as a "class 9" accident, should be considered in the analyses of the acceptability of returning TMI-1 to operation. 